\title{
Chaos and scale
}

\section{Emmanuel Paradis}

Institut des Sciences de l'Evolution, CC 64, Université Montpellier II, Place Eugène Bataillon, F-34095 Montpellier cedex 5, France

In a recent issue of TREE, Ruxton ${ }^{1}$ points out Csilling et al.'s results ${ }^{2}$ concerning the dynamic of a theoretical metapopulation. They observed that a set of linked chaotic populations exhibits neither chaotic temporal variations nor spatial structuring. In other studies, the opposite result was observed ${ }^{3-6}$. Ruxton argued that this contrast could be explained by the fact that the timescales of reproduction and dispersal are decoupled in Csilling et al.'s model.

I think the absence of chaos in Csilling et al.'s study could be explained by a particular feature of their model. The modelled metapopulation occupies a landscape composed of a finite number of patches. All local populations have the same chaotic dynamic. Dispersal occurs when the population density exceeds a fixed threshold according to a stepping-stone rule. A fixed fraction of the population emigrates to neighbouring patches. The dispersal process is reiterated until all populations are below the threshold, then reproduction starts again. Since a given population may receive dispersers from other patches, numerous dispersal steps may occur in what Csilling et al. call a 'migration avalanche'. The individuals dispersing out of the landscape are lost, so that all excess individuals are eliminated. Such a dispersal process is an efficient density- dependent regulating mechanism. It should be interpreted as an inhibition of reproduction in local populations as long as at least one population density exceeds the threshold. Such a mechanism does not seem biologically realistic, since it assumes that reproduction in a population is not only directly influenced by its own density but also by densities in the other populations. May and his collaborators ${ }^{7,8}$ pointed out a long time ago that chaos occurs when there is a discrepancy between the timescales of reproduction and its density-dependent inhibition. In Csilling et al.'s model, the local density-dependent mechanisms are outweighed by the dispersal mechanism.

As rightly emphasized by Ruxton, the relative timescales of reproduction and dispersal are critical for the dynamic of a metapopulation when local dynamics are taken into account. Two mechanisms of dispersal can be distinguished as extremes along a continuum - the steppingstone mechanism whereby the dispersers can reach only the neighbouring patches, and the 'common pool' of dispersers where the distance between patches is unimportant for the 
dispersers. This general scheme is further complicated by habitat selection when habitat quality varies among patches, and by the triggering mechanism of dispersal. The 'common pool' mechanism can have a strong stabilizing influence both on population and metapopulation dynamics even when local dynamics are strongly chaotic ${ }^{9,10}$. This is achieved providing the dispersal rate is sufficiently low. When dispersal rate increases, it 'homogenizes' the whole system (as seasonality can $\mathrm{do}^{11}$ ) so that chaos occurs. Stepping-stone dispersal contributes critically to metapopulation persistence ${ }^{3,4}$; however, its stabilizing influence on global dynamics is yet to be demonstrated under realistic biological assumptions $^{12}$. Both dispersal mechanisms could be important for the same species at different spatial or temporal scales.

\section{References}

1 Ruxton, G.D. (1995) Trends Ecol. Evol. 10, 141-142

2 Csilling, A., Janosi, I.M., Pasztor, G. and Scheuring, I. (1994) Phys. Rev. E50, 1083-1092

3 Comins, H.N., Hassell, M.P. and May, R.M. (1992) J. Anim. Ecol. 61, 735-748

4 Hassell, M.P., Comins, H.N. and May, R.M. (1994) Nature 370, 209-292

5 Pascual, M. (1993) Proc. R. Soc. London Ser. B 251, 1-7

6 Solé R.V., Valls, J. and Bascompte, J. (1992) Phys. Lett. A 166, 123-128

7 May, R.M. (1973) Ecology 54, 315-325

8 May, R.M., Conway, G.R., Hassell, M.P. and Southwood, T.R.E. (1974) J. Anim. Ecol. 43, $747-770$

9 Ruxton, G.D. (1994) Proc. R. Soc. London Ser. B 256, 189-193

10 Stone, L. (1993) Nature 365, 617-620

11 Grenfell, B.T., Balker, B.M. and Kleczkowski, A. (1995) Proc. R. Soc. London Ser. B 259, 97-103

12 Mackey, M. and Milton, J. (1995) Physica D 81, 1-17 